\title{
Adding Advanced Annotation Functionalities to an Existing Digital Library System
}

\author{
M. Agosti and N. Ferro
}

\begin{abstract}
This study presents a solution to add annotation functions to an available digital library management system. The solution is based on the integration of a specialized annotation service into an existing digital library, where the annotation service supports the creation, reading and listing of annotations together with the possibility of searching for digital objects within the digital library system also making use of the content of the annotations related to them.
\end{abstract}

\section{Introduction}

Annotations are not only a way of explaining and enriching an information resource with personal observations, but also a means of transmitting and sharing ideas to improve collaborative work practices. Furthermore, annotations allow users to naturally merge and link personal contents with the information resources provided by a Digital Library Management System (DLMS).

In this paper we discuss a service oriented approach to the development of an annotation service which can be integrated into distinct DLMS. The proposed approach is based on a formal model of the annotation, which we have developed and which formalizes the main concepts concerning annotations and the relationships among annotations and annotated information resources [1]. The formal model provides us with sound bases for designing and developing an annotation service which can be easily integrated into a DLMS. Indeed, a clear definition of the concepts related to the annotation allows us to separate the functionalities needed to manage, access, and search annotations, which constitute the core of an annotation service, from the functionalities needed to integrate such annotation service into a DLMS. Finally, the formal model constitutes the necessary ground work for the working out of search algorithms and query languages with a capacity of exploiting annotations.

Università di Padova, Dipartimento di Ingegneria dell’Informazione, Padua, Italy, agosti@dei.unipd.it, ferro@dei.unipd.it 
We present the integration of FAST, the Flexible Annotation Service Tool which has been conceived and developed at the Department of Information Engineering of the University of Padua [2, 3], within the DelosDLMS, a new-generation DLMS which has been developed in the context of the EU-funded project DELOS (a Network of Excellence on Digital Libraries) [4], and we show how advanced search functions based on annotations can be added to an existing DLMS.

\section{The Conceptual Structure of an Annotation Service}

Digital Library Management Systems usually offer some basic hypertext and browsing capabilities based on the available structured data, such as authors or references. But they do not normally provide users with advanced hypertext functionalities, where the information resources are linked on the basis of the semantics of their content and hypertext information retrieval functionalities are available. A relevant aspect of annotations is that they permit the construction over time of an useful hypertext [5], which relates pieces of information of personal interest, which are inserted by the final user, to the digital objects which are managed by the DLMS. In fact, the user annotations allow the creation of new relationships among existing digital objects by means of links that connect annotations together with existing objects. In addition, the hypertext between annotations and annotated objects can be exploited not only for providing alternative navigation and browsing capabilities, but also for offering advanced search functionalities, able to retrieve more and better ranked objects in response to a user query by also exploiting the annotations linked to them $[4,5]$.

Therefore, annotations can turn out to be an effective way of associating this kind of hypertext to a DLMS to enable the active and dynamic use of information resources. In addition, this hypertext can span and cross the boundaries of the single DLMS, if users need to interact with the information resources managed by diverse DLMS [3]. This latter possibility is quite innovative, because it offers the means for interconnecting various DLMS in a personalized and meaningful way for the end-user, and, as it has been highlighted in [6], this is a big challenge for the DLMS of the next generation. Figure 1 depicts a situation in which FAST manages the annotations that have been produced by two users and that are on documents that are managed by two DLMS.

\section{The DelosDLMS}

DelosDLMS is a prototype of the future next-generation DLMS, jointly developed by partners of the DELOS project. The goal of DelosDLMS is to combine text and audio-visual searching, to offer personalized browsing using new information visualization and relevance feedback tools, to allow retrieved information to be 


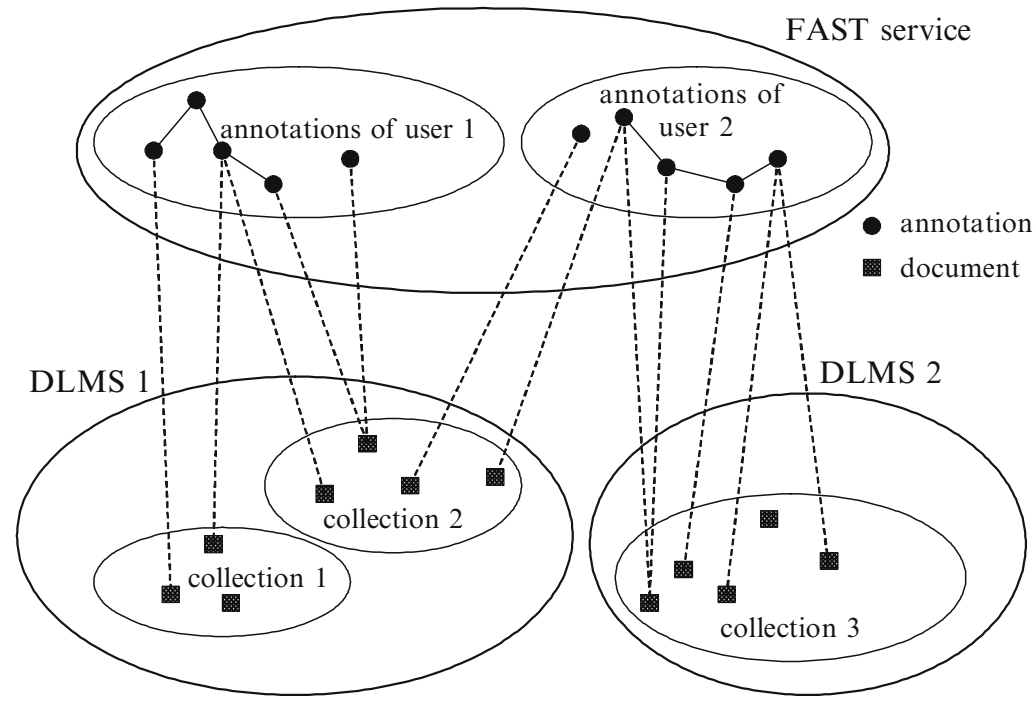

Fig. 1 Collections of users annotations managed by FAST and collections of annotated documents managed by different DLMS

annotated and processed, to integrate and process sensor data streams, and finally, from a systems engineering point of view, to be easily configured and adapted while being reliable and scalable.

The DelosDLMS prototype has been built by integrating digital library functionality provided by DELOS and non-DELOS partners into the OSIRIS/ISIS platform, a middleware environment developed by ETH Zürich and now being extended at the University of Basel. Open Service Infrastructure for Reliable and Integrated process Support (OSIRIS) has been chosen as basis for integration since it follows a serviceoriented architecture and thus allows to seamlessly add more functions which are provided behind a (Web) service interface [7]. Interactive SImilarity Search (ISIS) consists of a set of DL services that are built on top of OSIRIS. The ISIS services provide content-based retrieval of images, audio and video content, and the combination of any of these media types with sophisticated text retrieval [8]. DelosDLMS has the support for content-based retrieval of $3 \mathrm{D}$ objects $^{1}$ and advanced audio features. $^{2}$

\section{FAST Architecture}

FAST is a flexible system designed to support different architectural paradigms and a wide range of different DLMS. In order to achieve the desired flexibly:

\footnotetext{
${ }^{1}$ http://viplab.dsi.unifi.it/research/

${ }^{2}$ http://www.ifs.tuwien.ac.at/mir/
} 

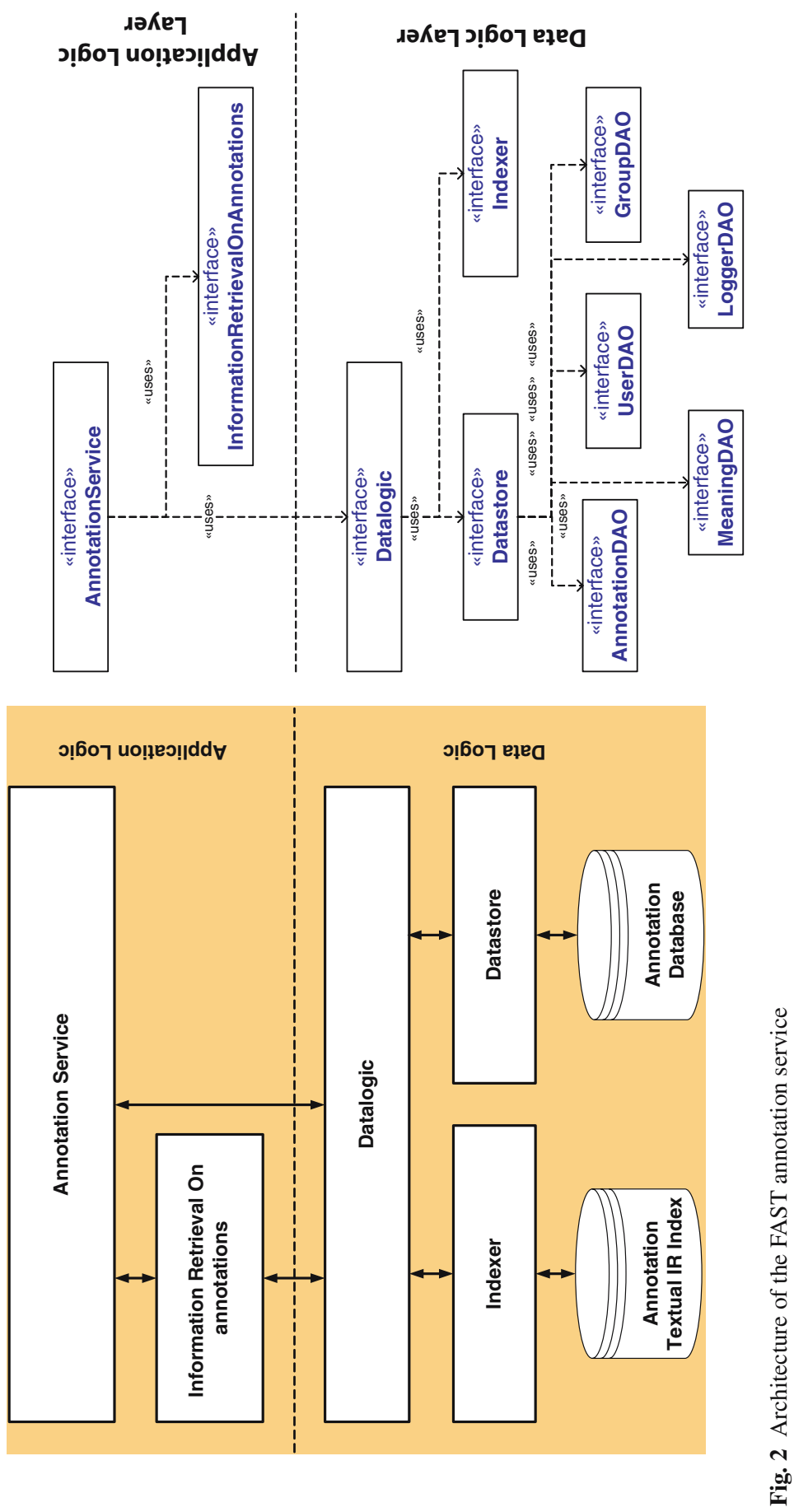
1. FAST is a stand-alone system, i.e., it is not part of any specific DLMS

2. The core functionalities of the annotation service are separated from the functionalities needed to integrate it into different DLMS

From an architectural point of view, FAST adopts a three-layers architecture - data, application and interface logic layers - and it is designed at a high level of abstraction in terms of abstract Application Program Interfaces (API) using an objectoriented approach. In this way, we can model the behavior and the functioning of FAST without worrying about the actual implementation of each component. Different alternative implementations of each component can be provided, still keeping a coherent view of the whole architecture of the FAST system.

Figure 2 shows the architecture and the components of FAST on the left side, while on the right side the main interfaces of the system are shown. In particular, the AnnotationService interface represents the entry point to the core functionalities of the annotation service.

Within FAST, annotations are composite multimedia objects, where each part of the annotation, called sign of annotation, has a well-defined and explicit semantics, called meaning of annotation. Annotations can annotate multiple parts of a given digital object and can relate this annotated digital object to various other ones, if this is of interest. Furthermore, once it has been created, an annotation is considered as a first class digital object, so that it can be annotated too, as it has depicted in Fig. 1.

From a functional point of view, FAST provides annotation management functionalities, such as creation, access, and so on. Furthermore it supports collaboration among users by introducing scopes of annotation and groups of users: annotations can be private, shared or public; if an annotation is shared, different groups of users can share it with different permissions, e.g., one group can only read the annotation while another can also modify it. Note that the annotation management engine ensures that validity constraints are complied with: for example, a private annotation cannot be annotated by a public annotation otherwise there would be a scope conflict, because the author of a private annotation can be allowed to see both public and private annotations, but another user can be only allowed to see public annotations. Finally, FAST offers advanced search functionalities based on annotations by exploiting annotations as a useful context in order to search and retrieve relevant documents for a user query $[9,10]$. The aim is to retrieve more documents that are relevant and to have them ranked in a way which is better than a system that does not makes use of annotations.

\section{Integration of FAST into the DelosDLMS}

The DelosDLMS supports two kinds of services: tightly-coupled services, i.e. services which implement the native protocol for service integration offered by OSIRIS, and loosely-coupled services, i.e. Web services that are integrated by using an OSIRIS component which acts as a gateway between the native OSIRIS protocol and the Simple Object Access Protocol (SOAP) protocol. 


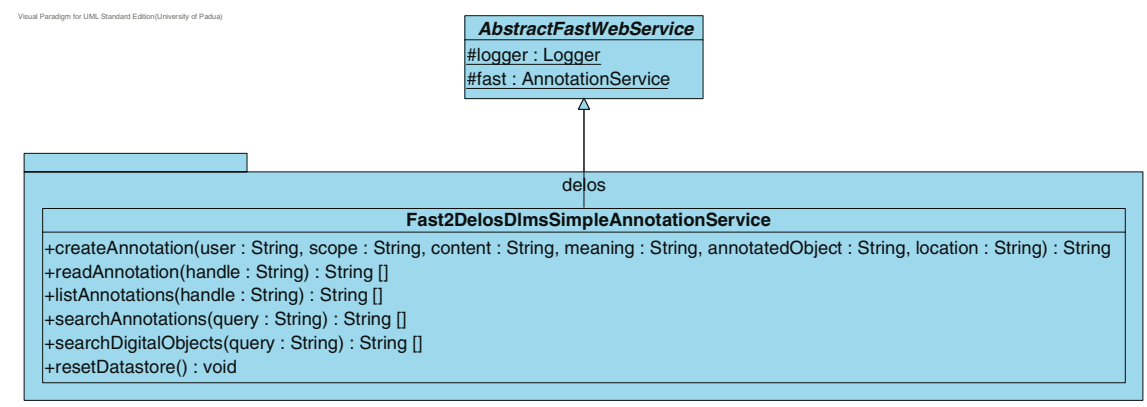

Fig. 3 UML class diagram of the designed Web service and its prototype implementation

FAST has been integrated into the DelosDLMS as a loosely-coupled service, i.e. as a Web service. First of all, the abstract class AbstractFastWebService has been developed. It wraps the AnnotationService component and provides the basic infrastructure for exposing its functionalities as a Web service; from this class, different concrete classes can be derived in order to integrate FAST as a Web service into different DLMS according to the specific characteristics of each DLMS. Secondly, the concrete class Fast2DelosDlmsSimpleAnnotationService has been derived from the abstract class AbstractFastWebService in order to provide basic annotation functionalities to the DelosDLMS.

Figure 3 depicts the Unified Modeling Language (UML) class diagram of the designed Web service where the functionalities made available are shown together with their input and output parameters. The functionalities made available by the developed Web service are:

- createAnnotation: Creates a new annotation, assuming that the annotation is constituted by only one textual sign and can be either public or private. In addition, a specific location of the annotated digital object can be specified, e.g. the upper left corner of an image

- readAnnotation: Reads an existing annotation with all related information

- listAnnotations: Returns a list of the annotation identifiers on a given digital object

- searchAnnotations: Performs a keyword-based search on the textual content of the annotations

- searchDigitalobjects: Performs a keyword-based search for digital objects on the basis of the content of their annotations by exploiting also the hypertext between digital objects and annotations

- resetDatastore: Completely resets the FAST datastore and its use is limited to the testing phase of the integration

The Fast2DelosDlmsSimpleAnnotationService has been implemented in Java ${ }^{3}$ by using the Apache Axis ${ }^{4}$ implementation of SOAP with the RPC/encoded binding style. Figure 4 shows the user interface where the results of an advanced search using annotations are reported: the grey results are retrieved using only the

\footnotetext{
${ }^{3}$ http: //java.sun.com/

${ }^{4}$ http://ws . apache.org/axis/
} 


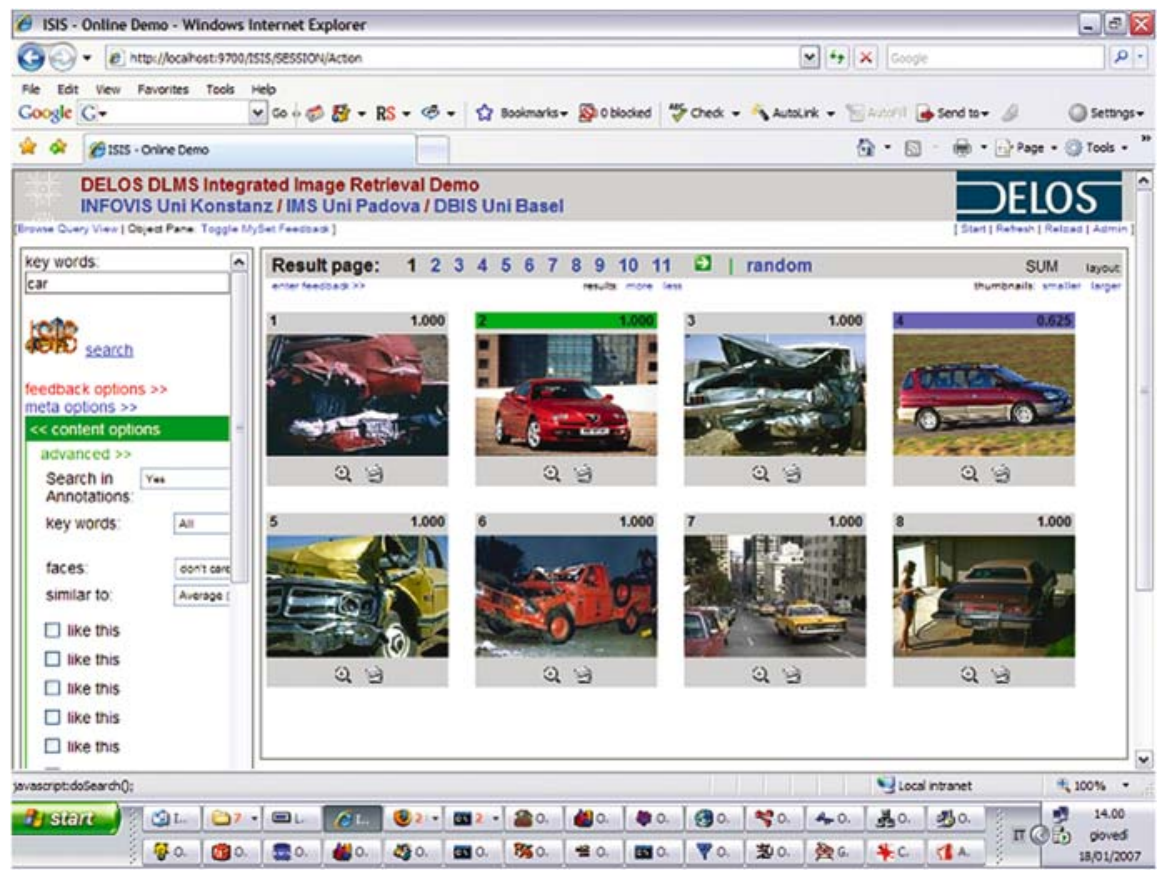

Fig. 4 DelosDLMS: advanced search functionalities based on annotations

metadata about the images; the green results are retrieved using only the annotations about the images; finally, the blue results are retrieved using both the metadata and the annotations about the images.

\section{Conclusions}

We have discussed the main features that an annotation service can offer to enhance usages of digital library systems and we have presented how the successful integration of the FAST annotation service in the DelosDLMS has been achieved.

Acknowledgments The work was partially supported by the DELOS Network of Excellence on Digital Libraries, as part of the Information Society Technologies (IST) Program of the European Commission (Contract G038-507618).

\section{References}

1. Agosti, M. and Ferro, N. (2008). A Formal Model of Annotations of Digital Content. ACM Transactions on Information Systems (TOIS), 26(1):1-55

2. Agosti, M. and Ferro, N. (2003). Annotations: Enriching a Digital Library. In T. Koch and I. T. Sølvberg, eds., Proceedings of the 7th European Conference on Research and 
Advanced Technology for Digital Libraries (ECDL 2003), pp. 88-100. LNCS 2769, Springer, Germany

3. Agosti, M. and Ferro, N. (2005). A System Architecture as a Support to a Flexible Annotation Service. In C. Türker, M. Agosti, and H.-J. Schek, eds., Peer-to-Peer, Grid, and ServiceOrientation in Digital Library Architectures: 6th Thematic Workshop of the EU Network of Excellence DELOS. Revised Selected Papers, pp. 147-166. LNCS 3664, Springer, Germany

4. Agosti, M., Berretti, S., Brettlecker, G., del Bimbo, A., Ferro, N., Fuhr, N., Keim, D., Klas, C.-P., Lidy, T., Milano, D., Norrie, M., Ranaldi, P., Rauber, A., Schek, H.-J., Schreck, T., Schuldt, H., Signer, B., and Springmann, M. (2007). DelosDLMS - the Integrated DELOS Digital Library Management System. In C. Thanos, F. Borri, L. Candela, eds., Digital Libraries: Research and Development. First International DELOS Conference. Revised Selected Papers, pp. 36-45. Lecture Notes in Computetr Science (LNCS) 4877, Springer, Germany

5. Agosti, M., Ferro, N., Frommholz, I., and Thiel, U. (2004). Annotations in Digital Libraries and Collaboratories - Facets, Models and Usage. In R. Heery and L. Lyon, eds., Proceedings of the 8th European Conference on Research and Advanced Technology for Digital Libraries (ECDL 2004), pp. 244-255. LNCS 3232, Springer, Germany

6. Ioannidis, Y., Maier, D., Abiteboul, S., Buneman, P., Davidson, S., Fox, E. A., Halevy, A., Knoblock, C., Rabitti, F., Schek, H.-J., and Weikum, G. (2005). Digital library informationtechnology infrastructures. Int. J Dig Libr, 5(4):266-274

7. Schuler, C., Schuldt, H., Türker, C., Weber, R., and Schek, H.-J. (2005). Peer-to-peer execution of (transactional) processes. Int. J Coop Inform Syst, 14:377-405

8. Mlivoncic, M., Schuler, C., and Türker, C. (2004). Hyperdatabase Infrastructure for Management and Search of Multimedia. In M. Agosti, H.-J. Schek, C. Türker, eds., Digital Library Architectures: Peer-to-Peer, Grid, and Service-Orientation, Pre-proceedings of the 6th Thematic Workshop of the EU Network of Excellence DELOS, pp. 25-36. Ed. Libreria Progetto, Italy

9. Agosti, M. and Ferro, N. (2005). Annotations as Context for Searching Documents. In F. Crestani and I. Ruthven, eds., Proceedings of the 5th International Conference on Conceptions of Library and Information Science (Colis 5), pp. 155-170. LNCS 3507, Springer, Germany

10. Agosti, M. and Ferro, N. (2006). Search Strategies for Finding Annotations and Annotated Documents: the FAST Service. In H. Legind Larsen, G. Pasi, D. Ortiz-Arroyo, T. Andreasen, and H. Christiansen, eds., Proceedings of the 7th International Conference on Flexible Query Answering Systems (FQAS 2006), pp. 270-281. LNAI 4027, Springer, Germany 